\title{
Temperament Style and Substance Abuse Characteristics
}

\author{
Melinda J. Henderson, Ph.D., ${ }^{1,3,4}$ Luke W. Galen, Ph.D., ${ }^{2}$ and \\ John W. DeLuca, Ph.D. ${ }^{1}$
}

The relationship of temperament to different patterns and types of alcohol abuse has received much attention over the last decade in order to provide clues to matching patients optimally to treatment strategies. The purpose of this study was to examine the relationship of temperament with a number of relevant substance abuse characteristics in a substance abusing population. One hundred forty-five male veterans were interviewed on their lifetime use of substances and on their psychiatric symptoms, problems associated with use, context, and family history of substance abuse. Subjects filled out the TPQ and the $M P Q$, which were subjected to factor analysis and revealed four factors: (1) Negative Affectivity/Impulsivity, (2) Positive Affectivity/Sociability, (3) Persistence/Achievement, and (4) Constraint. There was partial support for the hypotheses. Impulsivity was negatively correlated with age of onset and positively correlated with substance-related problems and a family history of substance abuse. Subjects with a history of depression scored significantly lower on the Positive Affectivity/ Sociability factor than those who had not experienced a significant depression. Individuals who used alone scored lower on this factor than those who used in social contexts. The temperament factors of Persistence/Achievement and Constraint were, for the most part, unrelated to substance abuse.

KEY WORDS: temperament; substance abuse; family history.

\section{INTRODUCTION}

Substance abuse is now viewed as a problem with multiple causes including biological/genetic, environmental, social, cultural, and many other influences (1-5). While alcohol and drug abuse are recognized increasingly as biopsychosocial disorders, there is still a tendency for clinicians, laypersons, and some researchers to

\footnotetext{
${ }^{1}$ Department of Psychiatry and Behavioral Neurosciences, Wayne State University, University Psychiatric Center, 2751 East Jefferson Avenue, Suite 200, Detroit, Michigan 48207.

${ }^{2}$ Alcohol Research Center, University of Michigan, Ann Arbor, Michigan.

${ }^{3}$ Addiction Research Institute, Wayne State University, Detroit, Michigan.

${ }^{4}$ To whom correspondence should be addressed.
}

61 
view substance abuse as a unitary "disease" process with multiple causes like many other diseases. Furthermore, there is an assumption that the disease eventually leads to the same course and set of outcomes. This is exemplified in the belief that all alcoholics go through the same stages and that all will "hit bottom" eventually. Much research over the last decade suggests that there is no single disorder but, rather, that there are many different forms of alcohol and drug abuse with distinct etiologies and patterns of abuse that suggest the necessity for different types of treatment (6-8).

Classification schemes have tended to overemphasize personality variables excluding the importance of biological, situational, and sociocultural influences (8) and excluding differences in social context and patterns of use and abuse (9). Although types of substance abusers are beginning to be elucidated, especially with regard to family history of alcohol abuse, temperament, and patterns of use and abuse, these factors are not often studied simultaneously.

There has been a tremendous amount of research investigating the relationship of temperament to substance abuse risk (10-14). The most consistent differentiating factor has been the presence or absence of conduct problems/antisocial behaviors which have been linked to a sensation-seeking temperament. The Type A/Type B classification (15) and the Type I/Type II distinction (1) have provided valuable heuristics for research, as multiple variables have shown a tendency to covary together including age at onset, number of alcohol-related problems, novelty-seeking, family history of alcoholism and mental illness, and other factors. In general, findings have indicated that the more severe type of the disorder is associated with a novelty-seeking temperament, a greater incidence of other psychopathology, an earlier age at onset, a higher paternal history of alcoholism, and a larger number of alcohol-related problems $(1,15)$. And the less severe form of the disorder has been shown to exhibit fewer of these characteristics (15). However, the hypothesis that the later-onset, less severe drinker type was more prone to a highly harm-avoidant and reward-dependent temperament (1) has not received much support (16-19). This lack of relationship may be due in part to the poor internal consistency of Reward Dependence, a scale on the Tridimensional Personality Questionnaire (TPQ) that was developed to test the hypothesis that different temperaments predisposed individuals to different types of alcohol abuse.

In recent years, the Type A/Type B distinction has been noted in the substance abuse population. Ball et al. (20) revealed Type A and Type B clusters in a population of cocaine abusers using family history of substance abuse, childhood disorders (conduct and attention deficit disorders), sensation-seeking, and age of onset of drug abuse as the initial clustering variables. Feingold et al. (21), using similar clustering variables with 521 inpatients, outpatients, and non-treatment seeking alcohol and drug dependent individuals, found that the Type A/Type B was generalizable for those with alcohol, cocaine, marijuana, and opiate dependence. In fact, the Type A/Type B distinction also seems to generalize across clinical settings, gender, and race (22). A recent article (19) reviewed numerous studies with alcohol and drug abusers who were administered the TPQ for making the Type 1/Type 2 distinction and concluded that the Novelty-Seeking scale has strong discriminant validity for both adult alcoholics and substance abusers. 
The overall purpose of this study was to extend and clarify further the relationship of temperament variables to the variables of age at onset, family history of alcoholism, social context of use, number of problems, and history of depression in a population of both alcohol and drug abusers. In order to examine more comprehensively relationships between temperament and characteristics of substance abusers, two measures of temperament, the TPQ and the Multidimensional Personality Questionnaire (MPQ), were included in the current study. In addition, the family history measure was extended to reflect all first and second degree relatives and to include the family history of drug and alcohol abuse. It was predicted that an impulsivity/novelty-seeking factor would emerge and be associated with a greater severity of problems as predicted by Babor's Type B classification. Specifically, impulsivity would be related to an earlier age at onset, a greater number of substancerelated problems, a greater likelihood of depression, a higher density of relatives with substance abuse problems, and a tendency to use in social contexts. A Sociability factor was predicted to emerge and to be negatively related to solitary use.

\section{METHOD}

\section{Subjects}

Subjects were 145 male inpatients in a 21- to 28-day chemical dependence treatment program at Allen Park Veterans Administration Hospital. Ages ranged from 21 to 59 years, with an average age of 39 years. Fifty-five percent of the sample was Caucasian and $39 \%$ was African-American. Eighty-three (57\%) subjects' primary drug of choice was alcohol, $39(27 \%)$ were primarily cocaine/crack users, 14 $(10 \%)$ used heroin primarily, and $2(1 \%)$ subjects classified themselves as using primarily marijuana. Six percent of the sample had two primary drugs of choice. Six subjects "reported" never using drugs and 115 subjects had experienced at least three drug-related problems independent of their alcohol-related problems.

\section{Measures}

Customary Drinking and Drug Use Record. The CDDR requires a structured interview entailing questions about the past 3 months of use, types of drugs used, physical and social context factors, drug preferences, drug/alcohol consumption lifetime history, problems related to substance abuse, and withdrawal symptoms. Three of the dependent variables in this study were derived from this questionnaire and were defined as follows: (1) context of use came from one question asking with whom the subject drank/used and was scored as either alone or with others. (2) Number of problems was obtained by asking subjects 17 questions related to problem areas which were either yes/no format or asked for the specific number of times the problem was experienced as a result of alcohol or drug use. Items from the CDDR include the DSMIII-R criteria for alcohol and substance dependence. For example, "Have you often taken alcohol/drugs in larger amounts or more often 
than you planned to?" The score for the number of problems for each subject was calculated according to their specified drug of choice. If their drug of choice was alcohol, the number of problems they reported as a result of alcohol use was used for this variable. If their drug of choice was any drug besides alcohol, their score on this measure was the number of problems they reported that occurred related to their drug use, (3) Age at onset of problematic use was obtained by asking subjects at what age they experienced all of their reported alcohol and drug use problems. The age at which they had experienced at least three alcohol/drug use problems was defined as their age of onset of problematic use because three criteria in the DSMIII-R are required to classify individuals as substance dependent.

Family Tree Questionnaire (23). Subjects were presented with a family tree diagram including self, siblings, parents, aunts, uncles, and grandparents. They were instructed to code each relative as one of the following: (1) an abstainer, (2) a social drinker, (3) a possible problem drinker, (4) a definite problem drinker, or (5) uncertain/do not know. Subjects completed another family tree diagram that required them to code relatives according to this same scheme for drug use/abuse status. Subjects were instructed to code only biological relatives. The Family Tree Questionnaire has yielded good test-retest reliability for both alcohol abusers and nonalcoholic populations. Kappa values for the various classifications ranged from .78 to .94 , with the lowest, yet acceptable values obtained with second-degree relatives.

The family expression of alcoholism (FEA) (24) score was utilized as a measure of family density of substance use problems. This study widened the scope to include family members with drug problems. Each family member that was coded either a 3 or a 4 for either alcohol or drug problems (a possible or definite problem drinker or a possible or definite drug abuser) was assigned a weight equivalent with firstdegree relatives weighted .5 and second-degree relatives weighted .25 . The FEA score was then computed by (1) summing the weights for the substance-abusing family members within each generation, (2) multiplying each sum by the ratio of the numbers of substance-abusing to the total number of family members in each generation, and (3) summing these scores across generations. Zucker et al. (1994) indicated that this measure is not a pure measure of biological risk because of child-rearing effects from the parents but that it is appropriate to regard it as a measure of genetic risk since many of the relatives who are not involved in the childhood environment contribute to the FEA score.

Schedule for Affective Disorders and Schizophrenia (SADS). The SADS is a structured interview which allows the trained interviewer to diagnose mood and thought disorders and has demonstrated good reliability (25). For this study, subjects were asked questions only from the depression section. This measure was used to classify subjects as positive or negative for a history of depression.

Tridimensional Personality Questionnaire (TPQ) (1). The TPQ consists of 100 true/false statements which describe attitudes, interests, opinions, and other personal feelings. It has three scales: (1) Novelty Seeking, (2) Harm Avoidance, and (3) Reward Dependence. Research $(16,17,26)$ has indicated that, although the TPQ scales have sufficient internal consistency, with alpha coefficients ranging from .71 to .89 , the $\mathrm{RD}$ scale was not a valid predictor of alcohol use or personality variables. 
The two subscales of RD have been found to be uncorrelated and seem to produce conceptual clarity resulting in two internally consistent scales $(17,27)$. Therefore, RD1 (Reward Dependence) and RD2 (Persistence) were scored as separate scales in this study.

Multidimensional Personality Questionnaire (MPQ) (28). The MPQ is a 300-item true/false questionnaire tapping attitudes, opinions, interests, and other qualities. It consists of three higher-order scales (Positive Affectivity, Negative Affectivity, and Constraint) representing 11 primary personality dimensions. The 11 dimensions have good internal consistencies ranging from .76 to .91 and the questionnaire has acceptable test-retest reliability (28).

\section{Procedure}

Charts were screened initially, and individuals with a current or past thought disorder diagnosis were excluded from the study. Three psychology graduate students who were informed of the general purpose of the study (not the specific hypotheses) were trained on the screening, interview procedures, and administration of the self-report inventories. A sign-up sheet was posted on the unit bulletin board explaining that volunteers were needed in a study examining the factors leading to drug and alcohol problems. In addition, patients were approached within 1 week of the beginning of their treatment and asked to participate in a study which would require them to fill out questionnaires and to answer questions about their substance use history. Those who agreed to participate read and signed an informed consent. The Wide Range Achievement Test-Revised (WRAT-R) (29) reading section was administered, and those who did not reach an eighth-grade reading level were excluded from the questionnaire part of the study and, thus, were excluded from the analyses.

Subjects met with the experimenter individually at least two times for up to 1 $\mathrm{hr}$ to answer questions from instruments that required one-on-one administration. In addition, subjects met in groups of one to six to fill out the personality questionnaires. Data were collected on each subject over a period of approximately 2 weeks while they remained in the hospital.

\section{Data Analyses}

Exploratory factor analysis using the SPSS 7.0 for Windows statistical package was conducted on the 10 scales of the MPQ and the 4 scales of the TPQ. Factor selection was based on eigenvalues greater than 1.0 as well as inspection of the scree plot. Next, Pearson product-moment correlations were performed on the four factor scores obtained with age at onset of problematic substance use, number of substance-related problems, and degree of family history of substance use problems. Finally, analyses of variance (ANOVAs) were conducted to determine if the temperament factor scores were significantly different based on the typical context of use (using alone vs. using with others) and history of depression. 


\section{RESULTS}

Preliminary analyses indicated that the age at onset of problematic use ranged from 12 to 51 years (mean $=24$ years), that $58 \%$ of the sample had a history of significant depressive symptomatology, and that approximately $49 \%$ of the subjects reported drinking/using alone. The family expression of alcoholism scores ranged from 0 to 5.06 (mean $=1.0$ ). Internal consistency coefficients ranged from .71 to .90 for the $10 \mathrm{MPQ}$ scales and from .65 to .89 for the 4 TPQ scales.

Exploratory factor analysis of the 14 scales of the TPQ and the MPQ revealed four factors: (1) Negative Affectivity/Impulsivity, (2) Positive Affectivity/Sociability, (3) Persistence/ Achievement, and (4) Constraint (see Table I for the factor loadings).

Correlation analyses indicated that Factor 1 (Negative Affectivity/Impulsivity) was negatively correlated with age at onset of problematic use $(r=-.17, p<.05)$ and positively correlated with number of problems associated with use $(r=.21, p$ $<.05)$ and with extent of family history of substance abuse $(r=.19, p<.05)$. Interestingly, Factor 3 (Persistence/Achievement) was also positively correlated with extent of family substance abuse involvement $(r=.20, p<.05)$. Factor 1 (Positive Affectivity/Sociability) and Factor 4 (Constraint) were unrelated to these three variables. There were no other significant correlations between all the factors and these three variables.

An analysis of variance (ANOVA) revealed that subjects with a history of depression scored significantly lower on Factor 2 (Positive Affectivity/Sociability) than individuals who had not been depressed $[F(1,139)=8.41, p<.01]$ (see Fig. 1). There were no significant differences on the other three factors between individuals with and without a history of depression.

An ANOVA indicated that subjects who preferred to use substances alone scored significantly lower on Factor 2 (Positive Affectivity/Sociability) than those

Table I. Factor Loadings for TPQ and MPQ Scales ${ }^{a}$

\begin{tabular}{lcccc}
\hline & Factor 1 & Factor 2 & Factor 3 & Factor 4 \\
\hline Control & -.65 & -.07 & .41 & .20 \\
Harm avoidance & -.28 & -.09 & -.15 & .61 \\
Traditionalism & .17 & .18 & .17 & .75 \\
Alienation & .70 & -.31 & .24 & .20 \\
Aggression & .76 & -.09 & .02 & -.12 \\
Stress reaction & .70 & -.46 & -.10 & .21 \\
Social potency & .43 & .58 & .21 & -.13 \\
Social closeness & -.15 & .83 & -.15 & .22 \\
Achievement & -.06 & .16 & .89 & -.03 \\
Well-being & -.06 & .67 & .37 & -.11 \\
Harm avoidance (HA) & .31 & -.69 & -.42 & .21 \\
Novelty seeking & .72 & .20 & -.32 & -.07 \\
Reward dependence (RD1) & -.03 & .66 & -.06 & .40 \\
Persistence (RD2) & .06 & .07 & .86 & .03 \\
\hline The first 10 scores are from the MPQ; the last 4 scales are from the TPQ. Factor \\
1, Negative Affectivity/Impulsivity; Factor 2, Positive Affectivity/Sociability; Factor \\
3, Persistence/ Achievement; Factor 4, Constraint. & &
\end{tabular}




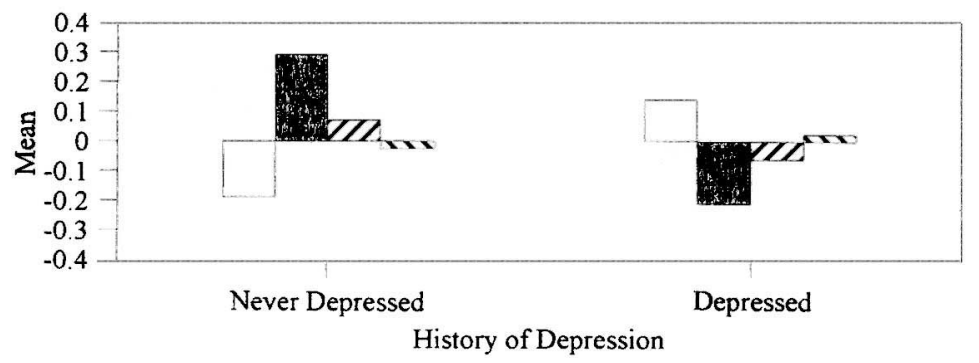

Neg. Affectivity/Impulsivity
Persistence/Achievement

Fig. 1. Factor score by history of depression.

who used primarily with others $[F(1,140)=12.70, p<.001]$ (see Fig. 2). There were no other significant differences on the remaining three factors for individuals who were solitary versus sociable users.

\section{DISCUSSION}

The results of this study suggest that substance-abusing individuals with impulsive temperaments and who exhibit negative affectivity are more likely to begin problematic use at an earlier age, to experience a larger number of substance-related problems, and to have a higher density of substance abuse in their relatives. These impulsive individuals, however, are not likely to use more frequently in social contexts or to have a history of depression. It is important to note that the factors that emerged from the two, three-factor instruments resulted in a splitting of the construct of novelty-seeking or behavioral activation. Specifically, impulsivity loaded on the first factor and sociability loaded on the second factor. Therefore, it is not surprising that context of use was unrelated to the first factor, but significantly related to the second factor which consisted of positive affectivity and sociability. Clearly, novelty-seeking does not reflect extraversion or sociability but instead a more pathological activity level (18).

The second factor that emerged, Positive Affectivity/Sociability, was negatively related to a history of depression and associated with solitary use. This finding is clearly consistent with research suggesting that the specific factor that defines depression is the absence of positive affectivity (rather than the presence of negative affectivity) and that positive affectivity is associated with extraversion and sociability (30). It is also important to note that the TPQ's scales of Harm Avoidance and Reward Dependence (RD 1) had the highest loadings on this factor and support Cloninger's notion of the Type 1 alcoholic as more introverted. These findings also suggest that this type of individual is prone to depression. 


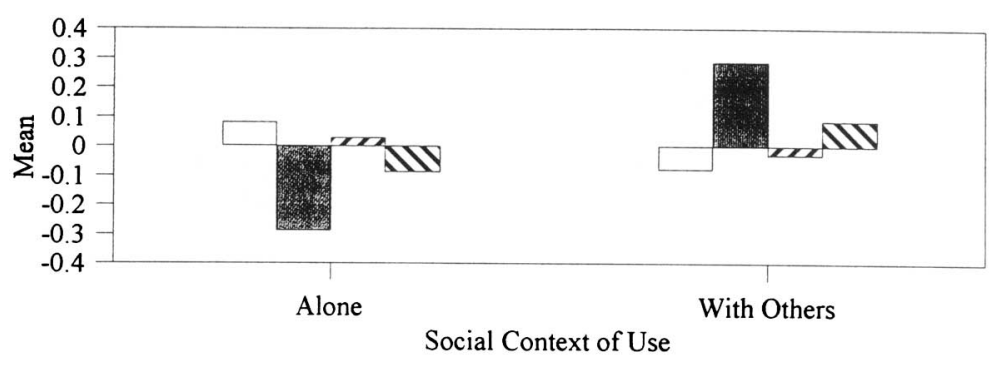

Neg. Affectivity/Impulsivity

Pos. Affectivity/Sociability

Persistence/Achievement

N Constraint

Fig. 2. Factor score by context of use.

Factor analysis of the TPQ and MPQ revealed two additional factors which in part subsume the two reward dependence scales of the TPQ and the MPQ constraint factor. Findings indicated that these temperament factors were for the most part unrelated to substance abuse variables. The fact that Persistence/Achievement was positively correlated with density of family history of substance abuse problems was not predicted and warrants replication. Overall, the lack of findings for the Persistence/Achievement and the Constraint factor is consistent with prior research using only the RD scale of the TPQ (16-18) and, thus, suggests that the lack of internal consistency of the TPQ's RD scale is not the only reason for the lack of relationship to substance abuse variables. Rather, the constructs of Persistence/Achievement and Constraint appear to be unimportant variables in distinguishing types of substance abusers in this sample. However, due to the restricted population of this study (a VA sample), one cannot rule out the possibility that the latter two factors may be related to substance abuse variables in a more heterogenous substance abusing sample. In fact, in a recent review of the use of the TPQ with substance abuse populations, Howard et al. (19) concluded that findings with Reward Dependence and Harm Avoidance have been inconsistent, and thus, larger samples and multiple statistical techniques are needed to clarify the dimensionality of the TPQ.

In sum, the findings of this study clearly support prior research indicating that negative affectivity/impulsivity is related to family history of substance abuse, earlier age at onset, and greater number of substance-related consequences. Sher (31) has suggested that there may be significant heterogeneity within this classification of Type 2 or Type B alcoholics. One possible distinction suggested by this study may be the presence or absence of comorbid depression. Treatment matching is indicated, as individuals with comorbid depression may need a more intensive treatment approach that addresses the depression with additional psychosocial and pharmacological approaches. Individuals who tend to be solitary users may need treatment that encourages socialization skills, whereas those who tend to use with others are likely to need a greater focus on developing new social networks and refusal skills. 
Research needs to continue to clarify the utility of examining other temperament characteristics relationships to substance abuse variables.

\section{REFERENCES}

1. Cloninger CR: Neurogenetic adaptive mechanisms in alcoholism. Science $1987 ; 23: 410-416$

2. Donovan JE, Jessor R, Costa FM: Syndrome of problem behavior in adolescence: A replication. $J$ Consult Clin Psychol 1988; 56:762-765

3. Marlatt GA, Baer JS, Donovan DM, Kivlahan DR: Addictive behaviors: Etiology and treatment. Annu Rev Psychol 1988; 39:223-252

4. Tarter RE: Are there inherited behavioral traits that predispose to substance abuse? J Consult Clin Psychol 1988; 56:189-196

5. Tarter RE, Kabene M, Escallier EA, Laird SB: Temperament deviation and risk for alcoholism. Alcohol Clin Exp Res 1990; 14:380-382

6. Blitz CC, Del Boca FK, Babor TF: Subtyping adolescents by vulnerability and risk factors. Paper presented at the Annual Meeting of the Research Society on Alcoholism, Washington, DC, 1996

7. Cloninger CR, Sigvardsson S, Gilligan SB, von Knorring A, Reich T, Bohman M: Genetic heterogeneity and the classification of alcoholism. Adv Alcohol Substance Abuse 1988; 7:3-16

8. Graham JR, Strenger VE: MMPI characteristics of alcoholics: A review. J Consult Clin Psychol 1988; 56:197-205

9. Hughes SO, Power TG, Frances DJ: Defining patterns of drinking in adolescence: A cluster analytic approach. J Stud Alcohol 1992; 53:161-169

10. Galen LW, Henderson MJ, Whitman RD: The utility of novelty-seeking, harm avoidance, and expectancy in the prediction of drinking. Addict Behav 1997; 22:93-106

11. Henderson MJ, Goldman MS, Coovert MD, Carnevalla N: Covariance structure models of expectancy. I Stud Alcohol 1994; 55:315-326

12. Ichiyama MA, Zucker RA, Fitzgerald HE, Bingham CR: Articulating subtype differences in self and relational experience among alcoholic men using structural analysis of social behavior. $J$ Consult Clin Psychol 1996; 64:1245-1254

13. Nathan PE: The addictive personality is the behavior of the addict. J Consult Clin Psychol 1988; 56:183-188

14. Tarter RE, Vanyukov 1994: Alcoholism: A developmental disorder. J Consult Clin Psychol 1994; 62:1096-1107

15. Babor TF, Hofmann M, Del Boca FK, Hesselbrock VM, Meyer RE, Dolinsky ZS, Rounsaville BJ: Types of alcoholics. I. Evidence for an empirically derived typology based on indicators of vulnerability and severity. Arch Gen Psychiatry 1992; 49:599-614

16. Cannon DS, Clark LA, Leeka JK, Keefe CK: A reanalysis of the Tridimensional Personality Questionnaire (TPQ) and its relation to the Cloninger's type 2 alcoholism. Psychol Assess 1993; 5:62-66

17. Waller NG, Lilienfeld SO, Tellegen A, Lykken DT: The Tridimensional Personality Questionnaire: Structural validity and comparison with the Multidimensional Personality Questionnaire. Multivar Behav Res 1991; 26:1-23

18. Wetzel RD, Knesvich MA, Brown SL, Wolff HA, Horn CJ, Cloninger CR: Correlates of the TPQ scales with selected MMPI scale;. Psychol Reports 1992; 71:1027-1038

19. Howard MO, Kivlahan D, Walker D: Cloninger's tridimensional theory of personality and psychopathology: Applications to substance use disorders. J Stud Alcohol 1997; 58:48-66

20. Ball SA, Carroll KM, Babor TF, Rounsaville BJ: Subtypes of cocaine abusers: Support for a Type A-Type B distinction. J Consult Clin Psychol 1995; 163:115-124

21. Feingold A, Ball SA, Kranzler HR, Rounsaville BJ: Generalizability of the Type A/Type B distinction across different psychoactive substances. Am J Drug Alcohol Abuse 1996; 22:449-46

22. Ball SA: Type A and Type B alcoholism: Applicability across subpopulations and treatment settings. Alcohol Health Res World 1996; 20:30-35

23. Mann RE, Sobell LC, Sobell MB, Pavan D: Reliability of a family tree questionnaire for assessing family history of alcohol problems. Drug Alcohol Depend 1985; 15:61-67

24. Zucker RA, Ellis DA, Fitzgerald, HE: Developmental evidence for at least two alcoholisms. Ann NY Acad Sci 1994; 708:134-136

25. Spitzer RL, Endicott J, Robins E: Research diagnostic criteria: Rationale and reliability. Arch Gen Psychiatry 1978; 135:773-782 
26. Earleywine M, Finn PR, Peterson JB, Pihl RO: Factor structure and correlates of the Tridimensional Personality Questionnaire. J Stud Alcohol 1992; 53:233-238

27. Svrakic DM, Whitehead C, Przybeck TR, Cloninger CR: Differential diagnosis of personality disorders by the seven-factor model of temperament and character. Arch Gen Psychiatry 1993; 50:991-999

28. Tellegen A: Brief manual for the Multidimensional Personality Questionnaire. Minneapolis, MN: 1982

29. Jastak S, Wilkinson GS: Wide Range Achievement Test-Revised (WRAT-R) manual. Wilmington, DE: Jastak Associates; 1984

30. Clark LA, Watson D: Tripartite model of anxiety and depression: Psychometric evidence and taxonomic implications. J Abnorm Psychol 1991; 100:316-336

31. Sher KJ: There are two kinds of alcoholism researchers: Those who believe in two types of alcoholism and those who don't. Addiction 1994; 89:1061 